\title{
A Fourier transform and convolution of Diamond operator
}

\section{Wanchak Satsanit}

Department of Mathematics, Faculty of Science, Maejo University, Chiang Mai, 50290, Thailand.

\begin{abstract}
In this paper, we define a new operator and give a sense of distribution theory to find the Fourier transform of new operator. It was found that the Fourier transform of new operator related to the Fourier transform of ultrahyperbolic operator and Diamond operator. And we also study the convolution products $\square^{\mathrm{k}} \delta * \square^{\mathrm{l}}$ and $\diamond^{\mathrm{k}} \delta * \diamond^{\mathrm{l}}$.
\end{abstract}

Keywords: Diamond operator, Fourier transform, hypersurface.

2010 MSC: 46F10, 46F12.

(C)2019 All rights reserved.

\section{Introduction}

Let $x=\left(x_{1}, x_{2}, \ldots, x_{n}\right)$ be a point of $n$ dimensional Euclidean space $\mathbb{R}^{n}$, and $G=G(m, x)$ defined by

$$
G=G(m, x)=\left(\sum_{i=1}^{p} x_{i}^{2}\right)^{m}-\left(\sum_{j=p+1}^{p+q} x_{j}^{2}\right)^{m} .
$$

The hypersurface $\mathrm{G}$ is due to Kananthai and Nonlaopon [7]. We observe that by putting $\mathrm{m}=1$ in (1.1) we obtain

$$
G(1, x)=\left(\sum_{i=1}^{p} x_{i}^{2}\right)-\left(\sum_{j=p+1}^{p+q} x_{j}^{2}\right)=P(x) .
$$

The quadratic form defined by (1.2) is by Gelfand and Shilop [3].

The hypersurface $G=0$ is a generalized of hypercone $P=0$ with a singular point of the origin.

Let $\varphi(x)$ be the $\mathbb{C}^{\infty}$ function with the compact support defined from $\mathbb{R}^{n}$ to $\mathbb{R}$. Nonlaopon and Aguirre [6] have studied $\mathrm{I}_{+}^{\lambda}$ where $\mathrm{I}_{+}^{\lambda}$ defined by

$$
\left(I_{+}^{\lambda}, \varphi\right)=\int_{I>0}(I)^{\lambda} \varphi(x) d x=\int_{0}^{\infty} u^{\lambda+\frac{n}{2 m}-1} \phi(\lambda, u) d x, \quad I=I(x)=(a r)^{2}-(b s)^{2},
$$

Email address: wanchack@gmail.com (Wanchak Satsanit)

doi: $10.22436 /$ jnsa.012.10.04

Received: 2017-07-20 Revised: 2019-02-27 Accepted: 2019-04-30 
where $a, b$ are real numbers

$$
\begin{aligned}
& r^{2}=x_{1}^{2}+x_{2}^{2}+\cdots+x_{p}^{2} \text { and } s^{2}=x_{p+1}^{2}+x_{p+2}^{2}+\cdots+x_{p+q}^{2} \\
& \phi(\lambda, u)=\frac{1}{4 m^{2}} \int_{0}^{1}(1-t)^{\lambda} t^{\frac{q}{2 m}-1} \varphi_{1}(u, u t) d t, \\
& \varphi_{1}(u, u t)=\int \varphi d \Omega_{p} d \Omega_{q} \text {, }
\end{aligned}
$$

$d \Omega_{p}$ and $d \Omega_{q}$ are elements of surface area on the unit sphere in $\mathbb{R}^{p}$ and $\mathbb{R}^{q}$, respectively.

Consequently, $\left(\mathrm{G}_{+}^{\lambda}\right)$ has two sets of singularities, namely

$$
\lambda=-1,-2, \ldots,-k, \ldots, \quad \text { and } \quad \lambda=-\frac{n}{2 m},-\frac{n}{2 m}-1, \ldots,-\frac{n}{2 m}-s, \ldots,
$$

where $k=1,2,3, \ldots$, and $s=0,1,2, \ldots$.

In 1996, for the first time, Kananthai [5] has introduced the operator $\diamond^{k}$ and named it as Diamond operator iterated $k$ times and is defined by

$$
\diamond^{k}=\left(\left(\sum_{i=1}^{p} \frac{\partial^{2}}{\partial x_{i}^{2}}\right)^{2}-\left(\sum_{j=p+1}^{p+q} \frac{\partial^{2}}{\partial x_{j}^{2}}\right)^{2}\right)^{k}, p+q=n .
$$

$n$ is the dimension of the space $\mathbb{R}^{n}$ for $x=\left(x_{1}, x_{2}, \ldots, x_{n}\right) \in \mathbb{R}^{n}$ and $k$ is a nonnegative integer. The operator $\diamond^{k}$ can be expressed as

$$
\diamond^{k}=\triangle^{k} \square^{k}=\square^{k} \triangle^{k}
$$

where $\triangle^{k}$ is the Laplace operator defined by

$$
\triangle^{k}=\left(\frac{\partial^{2}}{\partial x_{1}^{2}}+\frac{\partial^{2}}{\partial x_{2}^{2}}+\cdots+\frac{\partial^{2}}{\partial x_{n}^{2}}\right)^{k}
$$

and $\square^{k}$ is the ultrahyperbolic operator iterated $k$ times defined by

$$
\square^{k}=\left(\frac{\partial^{2}}{\partial x_{1}^{2}}+\frac{\partial^{2}}{\partial x_{2}^{2}}+\cdots+\frac{\partial^{2}}{\partial x_{p}^{2}}-\frac{\partial^{2}}{\partial x_{p+1}^{2}}-\frac{\partial^{2}}{\partial x_{p+2}^{2}}-\cdots-\frac{\partial^{2}}{\partial x_{p+q}^{2}}\right)^{k} .
$$

Kananthai [5] has shown that the convolution $(-1)^{k} R_{2 k}^{e}(x) * R_{2 k}^{H}(x)$ is an elementary solution of the operator $\diamond^{k}$. That is

$$
\diamond^{k}\left((-1)^{k} R_{2 k}^{e}(x) * R_{2 k}^{H}(x)\right)=\delta(x),
$$

where the symbol $*$ designate convolution of $\mathrm{R}_{\alpha}^{e}$ and $\mathrm{R}_{\beta}^{\mathrm{H}}$. The function $\mathrm{R}_{2 \mathrm{k}}^{e}(\mathrm{x})$ is the Euclidean Riesz kernel and defined by (2.1) for $\alpha=2 k$ and $R_{2 k}^{H}(x)$ is the ultrahyperbolic Riesz kernel defined by (2.4) for $\alpha=2 k$, $k$ is nonnegative integer and $\delta$ is the Dirac delta distribution.

Next, Aguirre and Kananthai [1] introduced the family $K_{\alpha, \beta}$ and is defined by

$$
\mathrm{K}_{\alpha, \beta}=\mathrm{K}_{\alpha, \beta}(x)=\mathrm{R}_{\alpha}^{\mathrm{e}} * \mathrm{R}_{\beta}^{\mathrm{H}} .
$$

They studied the properties of $\mathrm{K}_{\alpha, \beta}$ and the convolution product of $\mathrm{K}_{\alpha, \beta} * \mathrm{~K}_{\alpha^{\prime}, \beta^{\prime}}$.

Now, in this article we introduce $H=H_{p, q, k, s}(m, x)$ defined by

$$
H_{p, q, k, s}(m, x)=\frac{\Gamma\left(\frac{p}{2}+k\right)}{\Gamma\left(\frac{p}{2}+k+s\right)}\left(\sum_{i=1}^{p} x_{i}^{2}\right)^{m}-\frac{\Gamma\left(\frac{q}{2}+k\right)}{\Gamma\left(\frac{q}{2}+k+s\right)}\left(\sum_{j=p+1}^{p+q} x_{j}^{2}\right)^{m},
$$


where $s$ is nonnegative integer and $p+q=n$ is the dimension of the space. We observe if we put $s=0, m=1$ in (1.7) we obtain

$$
H_{p, q, k, 0}(1, x)=\left(\sum_{i=1}^{p} x_{i}^{2}\right)-\left(\sum_{j=p+1}^{p+q} x_{j}^{2}\right)=G(1, x)=P(x) .
$$

This quadratic form is due to Gelfand and Shilov [4].

Firstly, we give a sense to Fourier transform of $(H(p, q, s, x))^{\alpha}$. On the suitable condition of $s$ and $m$ we obtain the Fourier transform of $(H(p, q, s, x))^{\alpha}$ related to the Fourier of ultrahyperbolic operator due to Trione [3], and the Fourier transform of Diamond operator due to Kananthai [6].

Finally we introduce the new operator $\mathrm{E}_{\mathrm{k}, \mathrm{s}}$ defined by

$$
\left(\sum_{i=1}^{p} x_{i}^{2}\right)^{1-s}\left(\sum_{i=1}^{p} \frac{\partial^{2}}{\partial x_{i}^{2}}\right)^{k}-\left(\sum_{j=p+1}^{p+q} x_{i}^{2}\right)^{1-s}\left(\sum_{i=p+1}^{p+q} \frac{\partial^{2}}{\partial x_{j}^{2}}\right)^{k},
$$

if $s \geqslant 0,\left(x_{1}, x_{2}, \ldots, x_{p}\right) \neq(0,0, \ldots, 0)$ and $\left(x_{p+1}, x_{2}, \ldots, x_{p+q}\right) \neq(0,0, \ldots, 0)$. Then the following formula is valid

$$
\left\{\left(H_{p, q, k, s}(m, x)\right)^{l}\right\}^{\wedge}=(-1)^{(k+s-1) l}\left(\frac{2^{2 s} \Gamma(k+s)}{4 \Gamma(k+1)}\right)^{l} E_{k, s}^{l}(\delta),
$$

where $\left(H_{p, q, r, s}(m, x)\right)$ is defined by $(1.7)$ and the symbol $\wedge$ means the Fourier transform. We also consider convolution product

$$
\mathrm{E}_{\mathrm{k}, \mathrm{s}}^{\mathrm{r}} \delta * \mathrm{E}_{\mathrm{k}, \mathrm{s}}^{\mathrm{l}} \delta=\mathrm{E}_{\mathrm{k}, \mathrm{s}}^{\mathrm{r}+\mathrm{l}} \delta
$$

If we put $k=1$ and $s=1$ in (1.8) we obtain the convolution product

$$
\square^{\mathrm{r}} \delta * \square^{\mathrm{l}} \delta=\square^{\mathrm{r}+\mathrm{l}} \delta,
$$

and if we put $k=2$ and $s=1$ in (1.8) we obtain the convolution product

$$
\diamond^{\mathrm{r}} \delta * \diamond^{\mathrm{l} \delta}=\diamond^{\mathrm{r}+\mathrm{l}} \delta
$$

where the operator $\diamond$ is defined by (1.3). Before going that point the followings definitions and some important concepts are needed.

\section{Preliminaries}

Definition 2.1. Let $x=\left(x_{1}, x_{2}, \ldots, x_{n}\right)$ be a point of $\mathbb{R}^{n}$ and $|x|=x_{1}^{2}+x_{2}^{2}+\cdots+x_{n}^{2}$. The function $R_{\alpha}^{e}(x)$ denotes the elliptic kernel of Marcel Riesz and is defined by

$$
R_{\alpha}^{e}(x)=\frac{|x|^{\frac{\alpha-n}{2}}}{D_{n}(\alpha)}
$$

where

$$
\mathrm{D}_{n}(\alpha)=\frac{\pi^{\frac{n}{2}} 2^{\alpha} \Gamma\left(\frac{\alpha}{2}\right)}{\Gamma\left(\frac{n-\alpha}{2}\right)},
$$

where $\alpha$ is a complex parameter and $n$ is the dimension of $\mathbb{R}^{n}$.

The function $R_{\alpha}^{e}(x)$ is precisely the definition of elliptic kernel of Marcel Riesz [4] and the following is valid

$$
\mathrm{R}_{\alpha}^{e}(x) * \mathrm{R}_{\beta}^{e}(x)=\mathrm{R}_{\alpha+\beta}^{e}(x)
$$


for $\alpha+\beta \neq n+2 l, l=0,1,2, \ldots$ Putting $q=0$ in (2.1) we have

$$
{ }_{p} R_{\alpha}^{e}(x)=\frac{|x|_{p}^{\alpha-p}}{D_{p}(\alpha)}
$$

where

$$
|x|_{p}^{2}=x_{1}^{2}+x_{2}^{2}+\cdots+x_{p}^{2}
$$

and

$$
\mathrm{D}_{\mathrm{p}}(\alpha)=\frac{\pi^{\frac{\mathrm{p}}{2}} 2^{\alpha} \Gamma\left(\frac{\alpha}{2}\right)}{\Gamma\left(\frac{p-\alpha}{2}\right)} .
$$

Similarly, if we put $p=0$ we have

$$
{ }_{q} R_{\alpha}^{e}(x)=\frac{|x|_{p}^{\alpha-p}}{D_{p}(\alpha)}
$$

where

$$
|x|_{q}^{2}=x_{1}^{2}+x_{2}^{2}+\cdots+x_{q}^{2}
$$

and

$$
\mathrm{D}_{\mathrm{q}}(\alpha)=\frac{\pi^{\frac{\mathrm{q}}{2}} 2^{\alpha} \Gamma\left(\frac{\alpha}{2}\right)}{\Gamma\left(\frac{\mathrm{q}-\alpha}{2}\right)} .
$$

Definition 2.2. Let $x=\left(x_{1}, x_{2}, \ldots, x_{n}\right)$ be a point of the $n$-dimensional Euclidean space $\mathbb{R}^{n}$. Denote by

$$
v=x_{1}^{2}+x_{2}^{2}+\cdots+x_{p}^{2}-x_{p+1}^{2}-x_{p+2}^{2}-\cdots-x_{p+q}^{2}
$$

the nondegenerated quadratic form and $p+q=n$ is the dimension of the space $\mathbb{R}^{n}$. Let $\Gamma_{+}=\left\{x \in \mathbb{R}^{n}\right.$ : $x_{1}>0$ and $\left.u>0\right\}$ and $\bar{\Gamma}_{+}$denotes it closure. For any complex number $\beta$, define the function

$$
R_{\alpha}^{H}(v)= \begin{cases}\frac{v^{\frac{\alpha-n}{2}}}{C_{n}(\alpha)}, & \text { for } x \in \Gamma_{+}, \\ 0, & \text { for } x \notin \Gamma_{+},\end{cases}
$$

where the constant $C_{n}(\beta)$ is given by the formula

$$
\mathrm{C}_{n}(\alpha)=\frac{\pi^{\frac{n-1}{2}} \Gamma\left(\frac{2+\alpha-n}{2}\right) \Gamma\left(\frac{1-\alpha}{2}\right) \Gamma(\alpha)}{\Gamma\left(\frac{2+\alpha-p}{2}\right) \Gamma\left(\frac{p-\alpha}{2}\right)} .
$$

The function $R_{\alpha}^{H}(v)$ is called the ultrahyperbolic kernel of Marcel Riesz and was introduced by Nozaki [9].

It is well known that $R_{\alpha}^{\mathrm{H}}(v)$ is an ordinary function if $\operatorname{Re}(\alpha) \geqslant n$ and is a distribution of $\alpha$ if $\operatorname{Re}(\alpha)<n$. Let supp $R_{\alpha}^{\mathrm{H}}(v)$ denote the support of $R_{\alpha}^{\mathrm{H}}(v)$. Suppose

$$
\operatorname{supp}_{\alpha}^{\mathrm{H}}(v) \subset \bar{\Gamma}_{+},
$$

that is supp $R_{\alpha}^{\mathrm{H}}(v)$ is compact. By putting $p=1$ in (2.4), (2.5), and remembering the Legendre' s duplication formula of $\Gamma(z)$

$$
\Gamma(2 z)=2^{2 z-1} \pi^{-\frac{1}{2}} \Gamma(z) \Gamma\left(z+\frac{1}{2}\right)
$$

then (2.1) reduces to

$$
M_{\alpha}^{\mathrm{H}}(v)= \begin{cases}\frac{v^{\frac{\alpha-n}{2}}}{\mathrm{E}_{\mathrm{n}}(\alpha)}, & \text { for } x \in \Gamma_{+}, \\ 0, & \text { for } x \notin \Gamma_{+} .\end{cases}
$$


Here $v(x)=x_{1}^{2}-x_{2}^{2}-\cdots-x_{n}^{2}$ and

$$
E_{n}(\alpha)=2^{\alpha-1} \pi^{\frac{n-2}{2}} \Gamma\left(\frac{\alpha}{2}\right) \Gamma\left(\frac{\alpha-n-2}{2}\right) .
$$

Putting $p=0$ in (2.4) and (2.5) we have

where

$$
{ }_{\mathrm{q}} \mathrm{R}_{\alpha}^{\mathrm{H}}(v)=\frac{|v|_{\mathrm{q}}^{\frac{\alpha-n}{2}}}{\mathrm{C}_{\mathrm{q}}(\alpha)}
$$

$$
v_{q}^{\frac{\alpha-n}{2}}=\left(-\left(x_{p+1}^{2}+x_{p+2}^{2}+\cdots+x_{p+q}^{2}\right)\right)^{\frac{\alpha-n}{2}}
$$

and

$$
\mathrm{C}_{\mathrm{q}}(\alpha)=\frac{\pi^{\frac{\mathrm{q}-1}{2}} \Gamma\left(\frac{2+\alpha-\mathrm{q}}{2}\right) \Gamma\left(\frac{1-\alpha}{2}\right) \Gamma(\alpha)}{\Gamma\left(\frac{2+\alpha}{2}\right) \Gamma\left(\frac{-\alpha}{2}\right)} .
$$

Lemma 2.3. The function $R_{\alpha}^{e}(x)$ defined by (2.1) has the following properties

$$
R_{0}^{e}(x)=\delta(x), \quad R_{-2 k}^{e}(x)=(-1)^{k} \triangle^{k} \delta(x), \quad \Delta^{k} R_{\alpha-2 k}^{e}(x)=(-1)^{k} R_{\alpha-2 k}(x),
$$

where $\triangle^{k}$ is the Laplace operator iterated $\mathrm{k}$ times defined by (1.5).

Proof. The proof of this lemma is given by Trione [4].

On the other hand, the Fourier transform of $R_{\alpha}^{e}(x)$ and $\triangle^{k} \delta$ are given by the following formula

$$
\begin{aligned}
\left(R_{\alpha}^{e}(x)\right)^{\wedge} & =|y|_{n}^{-\alpha}, \\
\left(\triangle^{k} \delta\right)^{\wedge} & =(-1)^{k}|y|_{n}^{2 k},
\end{aligned}
$$

where

$$
|y|_{n}^{2}=y_{1}^{2}+y_{2}^{2}+\cdots+y_{n}^{2}
$$

Lemma 2.4. The function $\mathrm{R}_{\alpha}^{\mathrm{H}}(\mathrm{x})$ defined by (2.4) has the following properties

$$
\begin{aligned}
R_{0}^{H}(x) & =\delta(x), \\
R_{-2 k}^{H}(x) & =\square^{k} \delta(x), \\
\square^{k} R_{\alpha}^{H}(x) & =R_{\alpha-2 k}^{H}(x), \\
\square^{k} R_{2 k}^{H}(x) & =\delta(x),
\end{aligned}
$$

where $\square^{\mathrm{k}}$ is the ultrahyperbolic operator iterated $\mathrm{k}$ times defined by (1.3).

Proof. The proof of this lemma is given by Aguirre [10].

Lemma 2.5. The convolution of $\mathrm{R}_{\alpha}^{\mathrm{H}}(\mathrm{x})$

$$
\mathrm{R}_{\alpha}^{\mathrm{H}} * \mathrm{R}_{\beta}^{\mathrm{H}}=\frac{\cos \left(\frac{\alpha \pi}{2}\right) \cos \left(\frac{\beta \pi}{2}\right)}{\cos \left(\left(\frac{\alpha+\beta}{2}\right) \pi\right)} \mathrm{R}_{\alpha+\beta}^{\mathrm{H}}
$$

if $\mathrm{p}$ is even and

$$
\mathrm{R}_{\alpha}^{\mathrm{H}} * \mathrm{R}_{\beta}^{\mathrm{H}}=\mathrm{R}_{\alpha+\beta}^{\mathrm{H}}+\mathrm{T}_{\alpha+\beta}
$$

if $\mathrm{p}$ is odd, where $\mathrm{R}_{\alpha}^{\mathrm{H}}$ is defined by (2.6) and (2.7),

$$
T_{\alpha, \beta}=T_{\alpha, \beta}(u \pm i 0, n)=\frac{\frac{2 \pi i}{4} C\left(-\frac{\alpha-\beta}{2}\right)}{C\left(-\frac{\alpha}{2}\right) C\left(-\frac{\beta}{2}\right)}\left[H_{\alpha+\beta}^{+}-H_{\alpha+\beta}^{-}\right]
$$


and

$$
\begin{aligned}
\mathrm{C}(\mathrm{r}) & =\Gamma(\mathrm{r}) \Gamma(1-\mathrm{r}), \\
\mathrm{H}_{\mathrm{r}}^{ \pm} & =\mathrm{H}_{\mathrm{r}}(\mathrm{u} \pm i 0, \mathrm{n})=e^{\mp \frac{\mathrm{r} \pi}{2} i} e^{ \pm \frac{q \pi}{2}} \mathrm{a}\left(\frac{r}{2}\right)(u \pm i 0)^{\frac{r-n}{2}}, \\
\mathrm{a}\left(\frac{\mathrm{r}}{2}\right) & =\Gamma\left(\frac{\mathrm{n}-\mathrm{r}}{2}\right)\left[2^{r} \pi^{\frac{n}{2}} \Gamma\left(\frac{r}{2}\right)\right]^{-1}, \\
(u \pm i 0)^{\lambda} & =\lim _{\epsilon \rightarrow 0}\left(u+i \epsilon|x|^{2}\right)^{\lambda} .
\end{aligned}
$$

In particular $\mathrm{R}_{\alpha}^{\mathrm{H}} * \mathrm{R}_{-2 \mathrm{k}}^{\mathrm{H}}=\mathrm{R}^{\mathrm{H}-2 \mathrm{k}}$ and $\mathrm{R}_{\alpha}^{\mathrm{H}} * \mathrm{R}_{2 \mathrm{k}}^{\mathrm{H}}=\mathrm{R}^{\mathrm{H}+2 \mathrm{k}}$.

Proof. The proof of this lemma is given by Aguirre [2].

Lemma 2.6. The function $\mathrm{K}_{\alpha, \beta}$ defined by (1.6) has the following properties

$$
\begin{aligned}
\mathrm{K}_{0,0} & =\delta(x), \\
\mathrm{K}_{-2 \mathrm{k},-2 \mathrm{k}} & =(-1)^{\mathrm{k}} \diamond^{\left.\mathrm{k}_{\{} \delta\right\},} \\
\diamond^{\mathrm{k}}\left\{\mathrm{K}_{\alpha, \beta}\right\} & =(-1)^{\mathrm{k}} \mathrm{K}_{\alpha-2 \mathrm{k}, \beta-2 \mathrm{k} .}
\end{aligned}
$$

Proof. The proof of this lemma is given by Aguirre and Kananthai [1].

Definition 2.7. Let $K_{\alpha, \beta}(x)$ be a distributional family defined by

$$
\mathrm{K}_{\alpha, \beta}(\mathrm{x})=\mathrm{R}_{\alpha}^{\mathrm{e}}(\mathrm{x}) * \mathrm{R}_{\beta}^{\mathrm{H}}(\mathrm{x}) \quad \text { and } \quad \mathrm{K}_{\alpha, \beta}(\mathrm{x})=\mathbb{F}\left\{\mathrm{F}^{-1}\left\{\mathrm{R}_{\alpha}^{\mathrm{e}}(\mathrm{x})\right\} \cdot \mathrm{F}^{-1}\left\{\mathrm{R}_{\beta}^{\mathrm{H}}(\mathrm{x})\right\}\right\},
$$

where the functions $R_{\alpha}^{e}(x)$ and $R_{\beta}^{H}(x)$ are defined by (2.1) and (2.4), respectively. $\mathbb{F}$ is the Fourier transform and $\mathbb{F}^{-1}$ is the inverse Fourier transform. The distribution family $K_{\alpha, \beta}(x)$ exists and is in the space $\mathrm{O}_{c}^{\prime}$ of rapidly decreasing distributions and was introduced by Aguirre and Kananthai in [1].

Lemma 2.8. Let $\left(x_{1}, x_{2}, \ldots, x_{n}\right)$ be a point of the $n$ dimensional Euclidean space $\mathbb{R}^{n}$. Consider a non-degenerate quadratic form in $\mathrm{n}$ variables of the form

$$
\mathrm{P}=\mathrm{P}(\mathrm{x})=\mathrm{x}_{1}^{2}+\mathrm{x}_{2}^{2}+\cdots+\mathrm{x}_{\mathrm{p}}^{2}-\mathrm{x}_{\mathrm{p}+1}^{2}-\cdots-\mathrm{x}_{\mathrm{p}+\mathrm{q}}^{2}
$$

and $\square^{\mathrm{k}}$ is the ultrahyperbolic operator and defined by (1.5). The following formula is valid

$$
\mathrm{P}^{-\mathrm{m}} \square^{\mathrm{k}} \delta(\mathrm{x})=\square^{\mathrm{k}+\mathrm{m}} \delta,
$$

where $\mathrm{m}$ is a nonnegative integer and $\delta(\mathrm{x})$ is the Dirac delta distribution.

Proof. The proof of this lemma is given by Aguirre and Trione [2].

Lemma 2.9. Let $\alpha$ be a complex number and $s, k, m$, and $i$ being nonnegative integers and $M_{-2 m(\alpha-i),-2 m i}^{p, s}$ is defined by

$$
M_{-2 m(\alpha-i),-2 m i}^{p, q, k, s}=\left(a_{p, k, s}\right)^{\alpha-i}\left({ }^{p} R_{-2 m(\alpha-i)}^{e}\right) *\left(a_{q, k, s}\right)^{i}\left({ }^{q} R_{-2 m i}^{H}\right),
$$

then we obtain

$$
\left(M_{-2 m(\alpha-i),-2 m i}^{p, q, k, s}\right)^{\wedge}=\left(a_{p, k, s}\right)^{\alpha-i}\left(a_{q, k, s}\right)^{i}|y|_{p}^{2 m(\alpha-i)}|y|_{q}^{2 m i}
$$

Proof. From (1.4) we have

$$
\mathrm{K}_{\alpha, \beta}=\mathrm{R}_{\alpha, \beta}(x)=\mathrm{R}_{\alpha}^{e} * \mathrm{R}_{\beta}^{\mathrm{H}}
$$

and considering that the Fourier transform of $R_{\alpha, \beta}(x)=R_{\alpha}^{e} * R_{\beta}^{H}$ [7] we have

$$
\begin{aligned}
\left(K_{\alpha, \beta}\right)^{\wedge} & =\left(R_{\alpha}^{e}\right)^{\wedge}\left(R_{\beta}^{H}\right)^{\wedge} \\
\left(M_{-2 m(\alpha-i),-2 m i}^{p, q, k, s}\right)^{\wedge} & =\left(a_{p, k, s}\right)^{\alpha-i}\left({ }^{p} R_{-2 m(\alpha-i)}^{e}\right)^{\wedge}\left(a_{q, k, s}\right)^{i}\left({ }^{q} R_{-2 m i}^{H}\right)^{\wedge}
\end{aligned}
$$


where the symbol $\wedge$ we means the Fourier transform. Now using (2.8) we have

$$
\left(p_{R_{-2 m(\alpha-i)}^{e}}\right)^{\wedge}=|y|_{p}^{2 m(\alpha-i)}
$$

and using (2.10) we obtain

$$
\begin{aligned}
\left({ }^{\mathrm{q}} \mathrm{R}_{-2 m i}^{\mathrm{H}}\right)^{\wedge}=\left({ }_{\mathrm{q}} \square^{\mathrm{mi}} \delta\right)^{\wedge} & =\left\{\left(-\left(\frac{\partial^{2}}{\partial x_{\mathrm{p}+1}^{2}}+\frac{\partial^{2}}{\partial x_{\mathrm{p}+2}^{2}}+\cdots+\frac{\partial^{2}}{\partial x_{\mathrm{p}+\mathrm{q}}^{2}}\right)\right)^{\mathrm{mi}} \delta\right\}^{\wedge} \\
& =\left((-1)_{\mathrm{q}}^{\mathrm{mi}} \triangle^{\mathrm{mi}} \delta\right)^{\wedge}=(-1)^{2 m i}|y|_{\mathrm{q}}^{2 m i}=|y|_{\mathrm{q}}^{2 m i}
\end{aligned}
$$

Taking account (2.12) and (2.13) into (2.14) we obtain

$$
\left(M_{-2 m(\alpha-i),-2 m i}^{p, q}\right)^{\wedge}=\left(a_{p, k, s}\right)^{\alpha-i}\left(a_{q, k, s}\right)^{i}|y|_{p}^{2 m(\alpha-i)}|y|_{q}^{2 m i}
$$

That completes the proof.

\section{Main results}

Theorem 3.1. Let $\mathrm{E}_{\mathrm{s}, \mathrm{k}, \mathrm{m}}^{\alpha}$ be the distribution family and defined by

$$
E_{s, k, m}^{\alpha}=\sum_{i=0}^{\infty}\left(\begin{array}{c}
\alpha \\
i
\end{array}\right) M_{-2 m(\alpha-i),-2 m i}^{p, q, k, s}
$$

where

$$
\left(\begin{array}{c}
\alpha \\
i
\end{array}\right)=\frac{\Gamma(\alpha+i)}{i ! \Gamma(\alpha-i+1)}
$$

then the following is valid

$$
\left.\left(E_{s, k, m}^{\alpha}\right)^{\wedge}=\left(N_{p, q, k, s}(m, y)\right)\right)^{\alpha}
$$

where $\mathrm{N}_{\mathrm{p}, \mathrm{q}, \mathrm{k}, \mathrm{s}}(\mathrm{m}, \mathrm{y})>0$ and defined by (1.7) and

$$
f^{\wedge}=\int_{R^{n}} f(x) e^{-i(x, y} d x .
$$

If $\mathrm{f}$ is a distribution with compact support by [9], equation (3.3) can be written as

$$
f^{\wedge}=\left\langle f(x), e^{-i(x, y)}\right\rangle .
$$

Proof. We evaluate the Fourier transform of $E_{s, k, m}^{\alpha}$ and have to show that

$$
\left(\sum_{i=0}^{\infty}\left(\begin{array}{l}
\alpha \\
i
\end{array}\right) K_{-2 m(\alpha-i),-2 m i}^{p, q, k, s}\right)^{\wedge}=\sum_{i=0}^{\infty}\left(\begin{array}{l}
\alpha \\
i
\end{array}\right)\left(K_{-2 m(\alpha-i),-2 m i}^{p, q, k, s}\right)^{\wedge}
$$

Let $g_{i}$ be the sequence

$$
g_{j}=\sum_{i=0}^{\infty}\left(\begin{array}{c}
\alpha \\
i
\end{array}\right) K_{-2 m(\alpha-i),-2 m i^{\prime}}^{p, q, k, s} \quad g=E_{s, k, m}^{\alpha} .
$$

Since $K_{\alpha, \beta}$ is in the space $\mathrm{O}_{c}^{\prime}$, then $K_{-2 m(\alpha-i),-2 m i}^{p, q}$ is in the space $O_{c}^{\prime}$, we conclude that the term of the sequence $g_{j}$ is bounded in $\mathbb{R}^{n}$. Therefore the sequence converges in $\mathrm{O}_{c}^{\prime}$ to $g$ and by continuity of the Fourier transform we conclude that (3.3) is valid for all $\alpha$. 
By the above reason the following formula is valid

$$
\left(E_{s, k, m}^{\alpha}\right)^{\wedge}=\left(\sum_{i=0}^{\infty}\left(\begin{array}{c}
\alpha \\
i
\end{array}\right) M_{-2 m(\alpha-i),-2 m i}^{p, q, k, s}\right)^{\wedge}=\sum_{i=0}^{\infty}\left(\begin{array}{c}
\alpha \\
i
\end{array}\right)\left(M_{-2 m(\alpha-i),-2 m i}^{p, q, k, s}\right)^{\wedge} .
$$

By Lemma 2.5, we obtain

$$
\begin{aligned}
\left(E_{s, k, m}^{\alpha}\right)^{\wedge} & \left.=\sum_{i=0}^{\infty}\left(\begin{array}{c}
\alpha \\
i
\end{array}\right)\left(a_{p, k, s}\right)^{\alpha-i}\left(|y|_{p}^{2 m}\right)^{\alpha-i}\right)\left(a_{q, k, s}\right)^{i}\left(\left(|y|_{q}^{2 m}\right)^{i}\right) \\
& =\left(a_{p, k, s}|y|_{p}^{2 m}-a_{q, k, s}|y|_{q}^{2 m}\right)^{\alpha}=\left(N_{p, q, k, s}(m, y)\right)^{\alpha},
\end{aligned}
$$

where $|y|_{\mathfrak{n}}^{2}$ is defined by (2.9). Thus

$$
\left(N_{p, q, k, s}(m, y)\right)^{\alpha}=\sum_{i=0}^{\infty}\left(\begin{array}{c}
\alpha \\
i
\end{array}\right)\left(\left(a_{p, k, s}|y|_{p}^{2 m}\right)^{\alpha-i}\right)\left(\left(a_{q, k, s}|y|_{q}^{2 m}\right)^{i}\right) .
$$

That completes the proof.

Theorem 3.2. Let $\mathrm{L}_{\mathrm{k}, \mathrm{s}}$ be the operator defined by the following form

$$
L_{k, s}=\left(\sum_{i=1}^{p} x_{i}^{2}\right)^{1-s}\left(\sum_{i=1}^{p} \frac{\partial^{2}}{\partial x_{i}^{2}}\right)^{m}-\left(\sum_{j=p+1}^{p+q} x_{j}^{2}\right)^{1-s}\left(\sum_{j=p+1}^{p+q} \frac{\partial^{2}}{\partial x_{j}^{2}}\right)^{m},
$$

if $s \geqslant 0,\left(x_{1}, x_{2}, \ldots, x_{p}\right) \neq(0,0, \ldots, 0)$ and $\left(x_{p+1}, x_{2}, \ldots, x_{\mathfrak{p}+q}\right) \neq(0,0, \ldots, 0)$. Then the following formula is valid

$$
\left\{\left(\mathrm{H}_{\mathrm{p}, \mathrm{q}, \mathrm{k}, \mathrm{s}}(\mathrm{m}, \mathrm{x})\right)^{\mathrm{l}}\right\}^{\wedge}=(-1)^{(\mathrm{k}+\mathrm{s}-1) \mathrm{l}}\left(\frac{2^{2 \mathrm{~s}} \Gamma(\mathrm{k}+\mathrm{s})}{4 \Gamma(\mathrm{k}+1)}\right)^{\mathrm{l}} \mathrm{E}_{\mathrm{k}, \mathrm{s}}^{\mathrm{s}}(\delta),
$$

where $\left(\mathrm{H}_{\mathrm{p}, \mathrm{q}, \mathrm{r}, \mathrm{s}}(\mathrm{m}, \mathrm{x})\right)$ is defined by (1.7).

Proof. By Theorem 3.1 we obtain the Fourier transform of $\left(N_{p, q, k, s}(y, m)\right)^{\alpha}$ by mean of the following formula

$$
\left(\left(N_{p, q, k, s}(y, m)\right)^{l}\right)^{\wedge}=\sum_{i=1}^{\infty}\left(\begin{array}{c}
\alpha \\
i
\end{array}\right) M_{-2 m(\alpha-i),-2 m i}^{p, q, k, s} .
$$

Letting $\alpha=l$ in (3.1) and using (3.2) we have

$$
\left(\begin{array}{l}
\alpha \\
i
\end{array}\right)= \begin{cases}\left(\begin{array}{l}
l \\
i
\end{array}\right), & \text { if } \alpha=l \\
0, & \text { if } i>l\end{cases}
$$

By (3.4), we have

$$
\begin{aligned}
\left(\left(N_{p, q, k, s}(y, m)\right)^{l}\right)^{\wedge} & =\sum_{i=1}^{\infty}\left(\begin{array}{l}
l \\
i
\end{array}\right) N_{-2 m(l-i),-2 m i}^{p, q, k, s} \\
& =\sum_{i=1}^{\infty}\left(\begin{array}{l}
l \\
i
\end{array}\right)^{p}\left(a_{p, k, s}\right)^{l-i} R_{-2 m(l-i)}^{e} *\left(a_{q, k, s}\right)^{i} R_{-2 m i}^{H} \\
& =\sum_{i=1}^{\infty}\left(\begin{array}{l}
l \\
i
\end{array}\right)(-1)^{m(l-i)}\left(a_{p, k, s}\right)^{l-i}\left(\triangle_{p}^{m} \delta\right)^{l-i} *\left(a_{q, k, s}\right)^{i}\left(\square_{q}^{m} \delta\right)^{i} \\
& =\sum_{i=1}^{\infty}\left(\begin{array}{l}
l \\
i
\end{array}\right)(-1)^{m(l-i)}\left(a_{p, k, s} \triangle_{p}^{m} \delta\right)^{l-i} *\left(a_{q, k, s} \triangle_{q}^{m} \delta\right)^{i}
\end{aligned}
$$

By using the properties [5]

$$
\square^{\mathrm{k}}\left\{\triangle^{\mathrm{l}} \delta\right\} * \square^{\mathrm{l}}\left\{\triangle^{\mathrm{k}} \delta\right\}=\square^{\mathrm{k}+\mathrm{l}}\left\{\triangle^{\mathrm{k}+\mathrm{l}} \delta\right\}
$$


From (3.5) we obtain the formula

$$
\left(N_{p, q, k, s}(m, y)\right)^{\wedge}=(-1)^{m l}\left(a_{p, k, s} \triangle_{p}^{m}-a_{q, k, s} \triangle_{q}^{m}\right)^{l} \delta .
$$

By Lemma 2.8, we have

$$
\mathrm{P}^{-\mathrm{s}} \square^{\mathrm{l}} \delta(\mathrm{x})=\mathrm{C}(\mathrm{s}, \mathrm{m}, \mathrm{l}) \square^{\mathrm{l}+\mathrm{s}} \delta(\mathrm{x})
$$

if $s<\frac{n}{2}$ and

$$
\mathrm{C}(\mathrm{s}, \mathrm{n}, \mathrm{l})=\frac{\Gamma(\mathrm{l}+1) \Gamma\left(\frac{\mathrm{n}}{2}+\mathrm{l}\right)}{2^{2 \mathrm{~s}} \Gamma(\mathrm{l}+\mathrm{s}+1) \Gamma\left(\mathrm{s}+\frac{\mathrm{n}}{2}+\mathrm{l}\right)} .
$$

From (3.1) and using (3.1) we have

$$
\mathrm{P}^{1-\mathrm{s}} \square^{\mathrm{l}} \delta(\mathrm{x})=\mathrm{C}_{1}(\mathrm{~s}, \mathrm{~m}, \mathrm{l}) \square^{\mathrm{l}+\mathrm{s}-1} \delta(\mathrm{x})
$$

if $s<\frac{n}{2}$ and

$$
\mathrm{C}_{1}(\mathrm{~s}, \mathrm{n}, \mathrm{l})=4 \frac{\Gamma(\mathrm{l}+1) \Gamma\left(\frac{\mathrm{n}}{2}+\mathrm{l}\right)}{2^{2 \mathrm{~s}} \Gamma(\mathrm{l}+\mathrm{s}) \Gamma\left(\mathrm{s}-1+\frac{\mathrm{n}}{2}+l\right)} .
$$

Thus

$$
\left(|x|_{\mathfrak{p}}^{2}\right)^{1-s} \triangle_{\mathfrak{p}}^{k}\{\delta(x)\}=C_{1}(s, p, k) \triangle_{p}^{k+s} \delta(x)
$$

and

$$
\left(|x|_{q}^{2}\right)^{1-s} \triangle_{q}^{k}\{\delta(x)\}=C_{1}(s, q, k) \triangle_{q}^{k+s} \delta(x)
$$

if $s<\frac{p}{2}, s<\frac{q}{2}$. From (3.7) and (3.8) we have

$$
\begin{aligned}
\mathrm{L}_{k, s} \delta & =\left(\left(|x|_{p}^{2}\right)^{1-s} \triangle_{\mathfrak{p}}^{k}-\left(|x|_{\mathrm{q}}^{2}\right)^{1-s} \triangle_{\mathrm{q}}^{\mathrm{k}}\right) \delta \\
& =\left(\mathrm{C}_{1}(s, \mathrm{p}, \mathrm{k}) \triangle_{\mathrm{p}}^{\mathrm{k}+\mathrm{s}-1}-\mathrm{C}_{1}(\mathrm{~s}, \mathrm{q}, \mathrm{k}) \triangle_{\mathrm{q}}^{\mathrm{k}+\mathrm{s}-1}\right) \delta \\
& =\frac{4 \Gamma(\mathrm{k}+1)}{2^{2 s} \Gamma(\mathrm{k}+\mathrm{s})}\left(\mathrm{a}_{\mathrm{p}, \mathrm{k}, \mathrm{s}} \triangle_{\mathrm{p}}^{\mathrm{k}+\mathrm{s}-1}-\mathrm{a}_{\mathrm{q}, \mathrm{k}, \mathrm{s}} \triangle_{\mathrm{q}}^{\mathrm{k}+\mathrm{s}-1}\right) \delta
\end{aligned}
$$

if $s<\frac{p}{2}$ and $s<\frac{q}{2}$ where $|x|_{p}^{2}$ and $|x|_{q}^{2}$ are defined by (2.2) and (2.3), respectively. Therefore we arrive at the following formula

$$
\begin{aligned}
\left(\left(N_{p, q, k, s}(y, m)\right)^{l}\right)^{\wedge} & =(-1)^{s l} \sum_{i=1}^{\infty}\left(\begin{array}{l}
l \\
i
\end{array}\right)\left(\triangle_{p}^{m}\right)^{l-i}\left(\triangle_{q}^{m}\right)^{i} \delta \\
& =(-1)^{s l}\left(a_{p, s} \triangle_{p}^{s}-a_{q, s} \triangle_{q}^{s}\right)^{l} \\
& =(-1)^{(k+s-1) l}\left(a_{p, k, s} \triangle_{p}^{k+s-1}-a_{q, k, s} \triangle_{q}^{k+s-1}\right)^{l} \delta \\
& =(-1)^{(k+s-1) l} \frac{2^{2 s} \Gamma(k+s)}{4 \Gamma(k+1)}\left(L_{k, s}^{l}\{\delta\}\right) .
\end{aligned}
$$

We obtain the Fourier transform of $\left(\mathrm{L}_{\mathrm{k}, \mathrm{s}}^{\mathrm{l}}\{\delta\}\right)$

$$
\left(\mathrm{L}_{\mathrm{k}, \mathrm{s}}^{\mathrm{l}}\{\delta\}\right)^{\wedge}=\left(\frac{(-1)^{(\mathrm{k}+\mathrm{s}-1)} 4 \Gamma(\mathrm{k}+1)}{2^{2 \mathrm{~s}} \Gamma(\mathrm{k}+\mathrm{s})}\right)^{\mathrm{l}}\left(\mathrm{N}_{\mathrm{p}, \mathrm{q}, \mathrm{k}, \mathrm{s}}(\mathrm{m}, \mathrm{y})\right)^{\mathrm{l}} \text {. }
$$

Using (1.1)

$$
\left[\left(\sum_{i=1}^{p} x_{i}^{2}\right)^{1-s}\left(\sum_{i=1}^{p} \frac{\partial^{2}}{\partial x_{i}^{2}}\right)^{m}-\left(\sum_{j=p+1}^{p+q} x_{j}^{2}\right)^{1-s}\left(\sum_{j=p+1}^{p+q} \frac{\partial^{2}}{\partial x_{j}^{2}}\right)^{l} \delta\right]^{\wedge}
$$




$$
=\left(\frac{(-1)^{(k+s-1)} 4 \Gamma(k+1)}{2^{2 s} \Gamma(k+s)}\right)^{l} \cdot\left[\frac{\Gamma\left(\frac{p}{2}+k\right)}{\Gamma\left(\frac{p}{2}+k+s-1\right)}\left(\sum_{i=1}^{p} x_{i}^{2}\right)^{k}-\frac{\Gamma\left(\frac{q}{2}+k\right)}{\Gamma\left(\frac{q}{2}+k+s-1\right)}\left(\sum_{j=p+1}^{p+q} x_{j}^{2}\right)^{k}\right]^{l}
$$

if $s<\frac{p}{2}$ and $s<\frac{q}{2}$. In particular letting $s=1$ and $k=m$ in the above equation we obtain the formula

$$
\left[\left(\sum_{i=1}^{p} \frac{\partial^{2}}{\partial x_{i}^{2}}\right)^{m}-\left(\sum_{j=p+1}^{p+q} \frac{\partial^{2}}{\partial x_{j}^{2}}\right)^{l} \delta\right]^{\wedge}=(-1)^{m}\left[\left(\sum_{i=1}^{p} x_{i}^{2}\right)^{m}-\left(\sum_{j=p+1}^{p+q} x_{j}^{2}\right)^{m}\right]^{l}
$$

Moreover letting $\mathrm{m}=1$ in (3.9) and using (1.5) we obtain the following formula

$$
\left(\square^{\mathrm{l} \delta}\right)^{\wedge}=\left((-1)^{\mathrm{l}} \mathrm{P}(\mathrm{y})\right)^{\mathrm{l}}
$$

The formula (3.10) appears in [6].

Now using the formula (3.6) we give a sense to convolution of $E_{s, k, m}^{\alpha} * E_{s, k, m}^{\beta}$ for $\alpha$ and $\beta$ being complex numbers. In fact the distribution $\mathrm{K}_{\alpha, \beta}^{\mathrm{p}, \mathrm{q}}$ defined by (2.5) is in the space $\mathrm{O}_{\mathrm{c}}^{\prime}$ then $\mathrm{E}_{\mathrm{s}, \mathrm{k}, \mathrm{m}}^{\alpha} \in \mathrm{O}_{\mathrm{c}}^{\prime}$ and by [8] we have

$$
\begin{aligned}
\left(E_{s, k, m}^{\alpha} * E_{s, k, m}^{\beta}\right)^{\wedge}=\left(E_{s, k, m}^{\alpha}\right)^{\wedge} \cdot\left(E_{s, k, m}^{\beta}\right)^{\wedge} & =\left(N_{p, q, k, s}(m, y)\right)^{\alpha} \cdot\left(N_{p, q, k, s}(m, y)\right)^{\beta} \\
& =\left(N_{p, q, k, s}(m, y)\right)^{\alpha+\beta}=\left(E_{s, k, m}^{\alpha+\beta}\right)^{\wedge}
\end{aligned}
$$

Thus

$$
E_{s, k, m}^{\alpha} * E_{s, k, m}^{\beta}=E_{s, k, m}^{\alpha+\beta} .
$$

In particular putting $\alpha=r$ and $\beta=l$ in (3.11) we obtain

$$
\begin{aligned}
\mathrm{L}_{\mathrm{k}, \mathrm{s}}^{\mathrm{r}+\mathrm{l}} & =\left(\mathrm{C}_{1}(\mathrm{~s}, \mathrm{k})\right)^{\mathrm{k}+\mathrm{l}}\left(\left(\mathrm{N}_{\mathrm{p}, \mathrm{q}, \mathrm{k}, \mathrm{s}}(\mathrm{m}, \mathrm{y})\right)^{\mathrm{r}+\mathrm{l}}\right)^{\wedge} \\
& =\left(\mathrm{C}_{1}(\mathrm{~s}, \mathrm{k})\right)^{\mathrm{r}}\left(\left(\mathrm{N}_{\mathrm{p}, \mathrm{q}, \mathrm{k}, \mathrm{s}}(\mathrm{m}, \mathrm{y})\right)^{\mathrm{r}}\right)^{\wedge}\left(\mathrm{C}_{1}(\mathrm{~s}, \mathrm{k})\right)^{\mathrm{l}}\left(\left(\mathrm{N}_{\mathrm{p}, \mathrm{q}, \mathrm{k}, \mathrm{s}}(\mathrm{m}, \mathrm{y})\right)^{\mathrm{l}}\right)^{\wedge}=\mathrm{L}_{\mathrm{k}, \mathrm{s}}^{\mathrm{r}} \delta * \mathrm{~L}_{\mathrm{k}, \mathrm{s}}^{\mathrm{l}} \delta,
\end{aligned}
$$

where $C_{1}(s, k)=\frac{(-1)^{k+s-1} 4 \Gamma(k+1)}{2^{2 s} \Gamma(k+s)}$. Therefore we obtain the following formula

$$
\begin{aligned}
& {\left[\left(\sum_{i=1}^{p} x_{i}^{2}\right)^{1-s}\left(\sum_{i=1}^{p} \frac{\partial^{2}}{\partial x_{i}^{2}}\right)^{k}-\left(\sum_{j=p+1}^{p+q} x_{j}^{2}\right)^{1-s}\left(\sum_{j=p+1}^{p+q} \frac{\partial^{2}}{\partial x_{j}^{2}}\right)^{k}\right]^{r+l} \delta} \\
& =\left[\left(\sum_{i=1}^{p} x_{i}^{2}\right)^{1-s}\left(\sum_{i=1}^{p} \frac{\partial^{2}}{\partial x_{i}^{2}}\right)^{k}-\left(\sum_{j=p+1}^{p+q} x_{j}^{2}\right)^{1-s}\left(\sum_{j=p+1}^{p+q} \frac{\partial^{2}}{\partial x_{j}^{2}}\right)^{k}\right]^{r} \delta \\
& *\left[\left(\sum_{i=1}^{p} x_{i}^{2}\right)^{1-s}\left(\sum_{i=1}^{p} \frac{\partial^{2}}{\partial x_{i}^{2}}\right)^{k}-\left(\sum_{j=p+1}^{p+q} x_{j}^{2}\right)^{1-s}\left(\sum_{j=p+1}^{p+q} \frac{\partial^{2}}{\partial x_{j}^{2}}\right)^{k}\right]^{l} \delta .
\end{aligned}
$$

Putting $s=1, k=2$ in (3.12) we obtain

$$
\left[\left(\sum_{i=1}^{p} \frac{\partial^{2}}{\partial x_{i}^{2}}\right)^{2}-\left(\sum_{j=p+1}^{p+q} \frac{\partial^{2}}{\partial x_{j}^{2}}\right)^{2}\right]^{r+l} \delta=\left[\left(\sum_{i=1}^{p} \frac{\partial^{2}}{\partial x_{i}^{2}}\right)^{2}-\left(\sum_{j=p+1}^{p+q} \frac{\partial^{2}}{\partial x_{j}^{2}}\right)^{2}\right]^{r} \delta
$$




$$
*\left[\left(\sum_{i=1}^{p} \frac{\partial^{2}}{\partial x_{i}^{2}}\right)^{2}-\left(\sum_{j=p+1}^{p+q} \frac{\partial^{2}}{\partial x_{j}^{2}}\right)^{2}\right]^{l} \delta
$$

Thus

$$
\diamond^{k+l} \delta=\diamond^{k} \delta * \diamond^{l} \delta
$$

That completes the proof.

\section{Acknowledgment}

The authors would like to thank The Thailand Research Fund and Office of the higher Education Commission and Graduate School, Maejo University, Chiang Mai, Thailand for financial support and also Prof. Amnuay Kananthai Department of Mathematics, Chiang Mai University for the helpful discussion.

\section{References}

[1] M. A. Aguirre Tellez, A. Kananthai, On the convolution product of the Distributional Families related to the Diamond operator, Matematiche (Catania), 57 (2002), 39-48. 1, 2, 2.7

[2] M. A. Aguirre Tellez, S. E. Trione, The distribution Multiplicative product $\mathrm{P}_{ \pm}^{-\frac{s}{2}} \delta(x)$, Rev. Colombiana Mat., 27 (1993), 1-7. 2, 2

[3] I. M. Gel'fand, G. E. Shilov, Generalized Function, (Translated from the Russian by Eugene Saletan), Academic Press [Harcourt Brace Jovanovich, Publishers], New York-London, (1964). 1, 1

[4] A. González Dominguez, S. E. Trione, On the Laplace transform of retarded Lorentz invariant functions, Adv. in Math., 31 (1979), 51-62. 1, 2, 2

[5] A. Kananthai, On the Solution of the n-Dimensional Diamond Operator, Appl. Math. Comput., 88 (1997), 27-37. 1, 1, 3

[6] A. Kananthai, On the Fourier transform of the Diamond kernel of Marcel Riesz, Appl. Math. Comput., 101 (1999), 151-158. 1, 1, 3

[7] A. Kananthai, K. Nonlaopon, On the Residue of Generalized Function P ${ }^{\lambda}$, Thai J. Math., 1 (2003), 49-57. 1, 2

[8] K. Nonlaopon, M. Aguirre Tellez, The Residue of the Generalized function $\mathrm{P}_{+}^{\lambda}$ in hypercone $\mathrm{P}=(\mathrm{ar})^{2}-(\mathrm{br})^{2}$, (to appear). 3

[9] Y. Nozaki, On Riemann-Liouville integral of ultra-hyperbolic type, Kodai Math. Sem. Rep., 16 (1964), 69-87. 2.2, 3.1

[10] S. E. Trione, M. A. Aguirre Tellez, The distribution convolution products of Marcel Riesz's ultra-hyperbolic kernel, Ravista de la Union Mathematica Argentina, 39 (1995), 115-124. 2 